\title{
Thermal filters for the ATHENA X-IFU: ongoing activities towards the conceptual design
}

\author{
Marco Barbera $^{1,2}$, A. Argan ${ }^{3}$, E. Bozzo ${ }^{4}$, G. Branduardi-Raymont ${ }^{5}$, \\ A. Ciaravella ${ }^{2}$, A. Collura ${ }^{2}$, F. Cuttaia ${ }^{6}$, F. Gatti ${ }^{7}$, A. Jimenez Escobar ${ }^{2}$, \\ U. Lo Cicero ${ }^{2}$, S. Lotti ${ }^{3}$, C. Macculi ${ }^{3}$, T. Mineo ${ }^{8}$, F. Nuzzo ${ }^{1,2}$, S. Paltani',

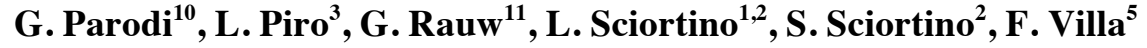 \\ 1 - UNIPA/Dipartimento di Fisica e Chimica, Palermo, Italy \\ 2 - INAF/Osservatorio Astronomico di Palermo G.S. Vaiana, Palermo, Italy \\ 3 - INAF/Istituto di Astrofisica e Planetologia Spaziale, Roma, Italy \\ 4- ISDC - Science data center for Astrophysics, Versoix, Switzerland \\ 5 - UCL/Dept. of Space and Climate Physics, MSSL, Dorking, Surrey, UK \\ 6 - INAF/Istituto di Astrofisica Spaziale e Fisica Cosmica, Bologna, Italy \\ 7 - UNIGE/Dipartimento di Fisica, Genova, Italy \\ 8 - INAF/Istituto di Astrofisica Spaziale e Fisica Cosmica, Palermo, Italy \\ 9 - Department of Astronomy, University of Geneva, Versoix, Switzerland \\ 10 - BCV progetti srl, Milano, Italy \\ 11 - UL/Dépt. Astrophysique, Géophysique, Océanographie, Liege, Belgium
}

\begin{abstract}
ATHENA is the L2 mission selected by ESA to pursue the science theme "Hot and Energetic Universe". One of the two focal plane instruments is the X-ray Integral Field Unit, an array of TES microcalorimeters operated at $\mathrm{T}<100 \mathrm{mK}$. To allow the X-ray photons focused by the telescope to reach the detector, windows have to be opened on the cryostat thermal shields. X-ray transparent filters need to be mounted on these open windows to attenuate the IR radiation from warm surfaces, to attenuate RF electromagnetic interferences on TES sensors and SQUID electronics, and to protect the detector from contamination. This paper reviews the ongoing activities driving the design of the X-IFU thermal filters.
\end{abstract}

Keywords X-ray Astronomy $\bullet$ ATHENA $\bullet$ X-IFU • Thermal Filters

\section{Introduction}

ATHENA[1] is an X-ray observatory, designed to address the "Hot and Energetic Universe" science theme[2], selected by ESA for L2 - the second Large-class mission within the Cosmic Vision Program (launch scheduled in 2028). One of the two instruments at the focal plane of the large area telescope[3] is the X-ray Integral Field Unit (X-IFU), an array of transition edge sensor (TES) microcalorimeters operating at temperatures $<100 \mathrm{mK}$, 
providing high spectral resolution $\left(\Delta \mathrm{E}_{\mathrm{FWHM}}=2.5 \mathrm{eV}\right.$ at $\left.\mathrm{E}<7 \mathrm{keV}\right)$ and imaging in the energy range $0.2-12 \mathrm{keV}$, over a 5 ' diameter field of view[4].

To allow the X-ray photons focused by the telescope to reach the detector, windows are opened on the cryostat shields. X-ray transparent filters need to be mounted on such shields to reduce IR radiation heat load and to prevent degradation of the energy resolution by photon shot noise[5]. The development of the thermal filters (TF) contains many technical challenges: small thickness for high transparency at soft X-rays, large size to cover the FOV, complex interface with the cryostat and focal plane assembly, detector protection from contamination, RF EMI attenuation at the telemetry X-band. For these reasons, the design, verification, and calibration of the TF are critical tasks in the ATHENA X-IFU implementation.

\section{Filter Design and Performance Modeling}

Previous space experiments with doped-silicon X-ray micro-calorimeters, have used TF based on al coated polyimide[6-10]. The ATHENA X-IFU has similar requirements on IR attenuation, however, the use of TES technology and SQUID based electronics put an additional requirement on shielding RF EMI from the spacecraft operation and telemetry. Based on such experience and new requirements on RF shielding, we baseline polyimide and aluminum for all $\mathrm{TF}$ with metal meshes on the two larger diameter filters. Fig. 1 left panel shows the modeled UV-VIS-IR transmission[5] for a set of five filters with a total of $225 \mathrm{~nm}$ polyimide and $150 \mathrm{~nm}$ aluminum. Upon our experience[11,12], we have assumed that $5 \mathrm{~nm}$ of oxide build up on each side of the Al coating and become transparent in the UV/Vis/IR.
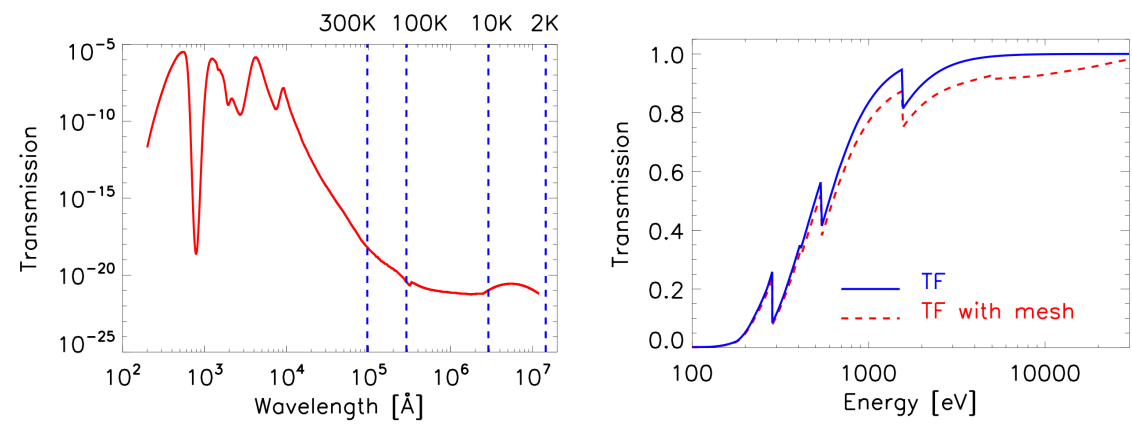

Fig. $1 \mathrm{UV} / \mathrm{VIS} / \mathrm{IR}$ transmission (left) for a set of five TF with a total of $225 \mathrm{~nm}$ polyimide and $150 \mathrm{~nm} \mathrm{Al.} \mathrm{X-ray} \mathrm{transmission} \mathrm{(right)} \mathrm{for} \mathrm{the} \mathrm{same} \mathrm{set} \mathrm{of} \mathrm{TF} \mathrm{without} \mathrm{meshes} \mathrm{(solid}$ line) and with a $96 \%$ open area Ti mesh on two filters (dashed line). Color figure online

The oxygen in the aluminum oxide $\left(\sim 1.9 \mu \mathrm{g} / \mathrm{cm}^{2}\right.$ per filter $)$ affects also the $\mathrm{X}$-ray transmission, it is indeed more than the oxygen present in $45 \mathrm{~nm}$ of 


\section{Thermal filters for the ATHENA X-IFU}

polyimide $\left(\sim 1.4 \mu \mathrm{g} / \mathrm{cm}^{2}\right)$. Fig. 1 right panel shows the modeled X-ray transmission for the same set of TF including the effect of aluminum oxide.

\subsection{Thermal Modeling}

Fig. 2 shows the TF configuration inside the cryostat adopted for the thermal modeling performed with Comsol Multiphysics ${ }^{\circledR}$ (not in scale).

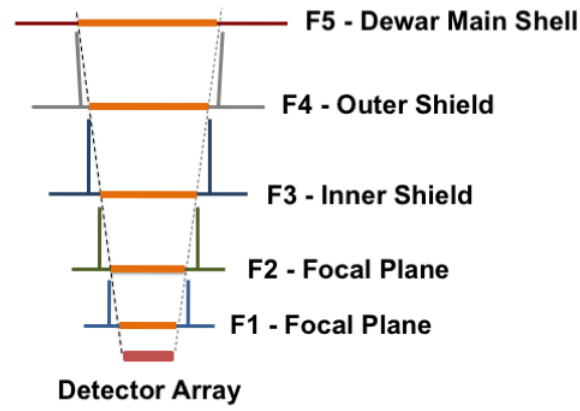

Fig. 2 Schematic TF configuration adopted in the thermal modeling (not in scale). The baffles, attached and thermally anchored to the shields, follow the telescope aperture (dashed line). Shield temperatures, filter diameters, and filter distances from the detector are reported in Table 1. Color figure online.

In the simulation, we have taken into account the al layer of each filter and the metal meshes in the two larger filters, while have neglected polyimide since it has a thermal conductivity much lower than al. Table 1 column 3 shows the derived equilibrium temperatures at the center of the five TF.

Table 1: Radiation power on the detector array and energy resolution degradation due to photon shot noise from the thermal radiation of the filters. For each filter we report: shield temperature, central filter temperature, distance from the array $(\mathrm{Z})$, and diameter (D).

\begin{tabular}{|c|c|c|c|c|c|c|}
\hline & $\begin{array}{c}\mathrm{T}_{\text {SHIELD }} \\
{[\mathrm{K}]}\end{array}$ & $\begin{array}{c}\mathrm{T}_{\text {FLTER }} \\
{[\mathrm{K}]}\end{array}$ & $\begin{array}{c}\mathrm{Z} \\
{[\mathrm{mm}]}\end{array}$ & $\begin{array}{c}\mathrm{D} \\
{[\mathrm{mm}]}\end{array}$ & $\begin{array}{c}\text { Power } \\
{[\mathrm{fW}]}\end{array}$ & $\begin{array}{c}\Delta E_{F W H M} \\
{[\mathrm{eV}]}\end{array}$ \\
\hline $\mathrm{F} 1$ & 0.05 & 0.87 & 10 & 24 & 8033 & 0.195 \\
$\mathrm{~F} 2$ & 2.0 & 2.1 & 60 & 36 & 4.446 & 0.007 \\
$\mathrm{~F} 3$ & 10.0 & 10.4 & 90 & 44 & 0.286 & 0.004 \\
F4 & 100.0 & 183 & 170 & 64 & 7.225 & 0.128 \\
F5 & 300.0 & 255 & 200 & 72 & 0.092 & 0.016 \\
\hline Total & & \multicolumn{7}{|c|}{} & $\mathbf{8 0 4 5}$ & $\mathbf{0 . 2 3 4}$ \\
\hline
\end{tabular}

\subsection{Radiation Heat Load and Photon Shot Noise}

Adopting the temperature profile derived from the thermal modeling and assuming that filters are tilted by $1^{\circ}$ in alternate directions to reduce multiple reflections, we have calculated the total radiation power onto the full detector array and the energy resolution degradation due to photon shot noise (Table 1), according to the recipe in [5]. If the filters are not tilted and they are perfectly flat, the multiple reflections increases the radiation power onto the detector and the energy resolution degrades to $>10 \mathrm{eV}$. 


\subsection{Structural Analysis}

Since the vibration levels of the X-IFU FPA and cryostat aperture cylinder are not yet known, we performed a preliminary structural analysis under 10 mbar static pressure (qualification static load for the XMM-Newton EPIC filters) on the mechanically more challenging larger filter $(D=72 \mathrm{~mm})$ where we foresee the use of a reinforcing mesh. Different parameters, namely: mesh material, geometry, pitch $(\mathrm{P})$, bar width $(\mathrm{BW})$, and thickness $(\mathrm{T})$ have been investigated. The currently most promising mesh consists of two Ti alloy Ti6A14V layers with honeycomb geometry: 1) a structural layer with $\mathrm{P}=5 \mathrm{~mm}, \mathrm{BW}=50 \mu \mathrm{m}, \mathrm{T}=250 \mu \mathrm{m}$, and 2) an $\mathrm{RF}$ attenuator layer with $\mathrm{P}=1$ $\mathrm{mm}, \mathrm{BW}=10 \mu \mathrm{m}, \mathrm{T}=30 \mu \mathrm{m}$. The blocking factor of such mesh is nearly $4 \%$. The calculated maximum tensile stress on the structural mesh and RF attenuator are $560 \mathrm{MPa}$ and $470 \mathrm{MPa}$, respectively, both values well within the ultimate tensile strength of the material $(950 \mathrm{MPa})$. The maximum tensile stress on a polyimide film $45 \mathrm{~nm}$ thick attached to such mesh is 140 $\mathrm{MPa}$ also well within the ultimate tensile strength of the material (310 $\mathrm{MPa})$.

\subsection{Radio Frequency EMI attenuation}

The telemetry uplink/downlink signals entering the instrument FOV by direct illumination or diffraction from satellite and instrument structures can cause EMI on TES detectors and SQUID electronics. A preliminary Electro Magnetic FEM (HFSS software by Ansys Corp.) was implemented with a 60 $\mathrm{mm}$ length standard WR-90 X-band waveguide, to evaluate the attenuation from conductive meshes in the $\mathrm{X}$-band frequency range $[8.2 \div 12.4 \mathrm{GHz}]$. The mesh geometry (e.g. honeycomb, square) is irrelevant to first order with respect to parameters such as mesh pitch, bar width, and thickness.

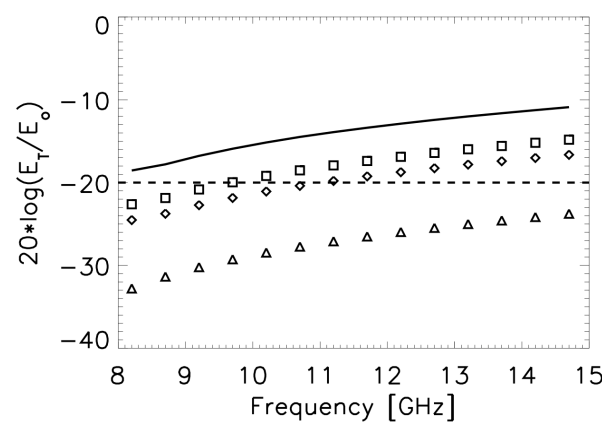

Fig. 3 Electric field attenuation vs. frequency for meshes with different pitches (P) and bar widths (BW) (equal to the mesh thickness), namely:

$$
\begin{aligned}
& \text { Solid line: } \quad \mathrm{P}=2 \mathrm{~mm}, \mathrm{BW}=20 \mu \mathrm{m} \\
& \text { Squares: } \mathrm{P}=1 \mathrm{~mm}, \mathrm{BW}=10 \mu \mathrm{m} \\
& \text { Diamonds: } \mathrm{P}=1 \mathrm{~mm}, \mathrm{BW}=20 \mu \mathrm{m} \\
& \text { Triangles: } \mathrm{P}=1 \mathrm{~mm}, \mathrm{BW}=100 \mu \mathrm{m} \\
& \text { The horizontal dashed line } \\
& \text { corresponds to a factor } 10 \text { attenuation. }
\end{aligned}
$$

Fig. 3 shows the electric field attenuation vs. frequency for a mesh with different pitches and bar widths (equal to the mesh thickness). The use of a 


\section{Thermal filters for the ATHENA X-IFU}

mesh with $1 \mathrm{~mm}$ pitch, and $10 \mu \mathrm{m}$ bar width on the two larger diameter TF should provide four orders of magnitude attenuation in RF power at $10 \mathrm{GHz}$.

\section{Optical Load from Astrophysical Sources}

The optical load from the bright UV/VIS counterparts of X-ray sources such as massive stars, AGN's in outburst, Mars, etc., can degrade the X-IFU energy resolution by photon shot noise. Fig. 4 (solid lines) shows the calculated X-IFU energy resolution degradation vs. visual magnitude for massive stars in the $T_{\text {eff }}$ range $15000 \div 45000 \mathrm{~K}$, assuming UV/VIS stellar spectra attenuation only by the TF. Even the faintest hot stars would have a non negligible degradation of the spectral resolution $\left(\Delta \mathrm{E}_{\mathrm{FWHM}}>0.2 \mathrm{eV}\right)$. On the other hand, the X-IFU capability to observe hot stars as bright as $\mathrm{m}_{\mathrm{v}}=2$ is mandatory to probe the dynamics of stellar winds in O-B stars [13]. Fig. 4 (dashed lines) shows the same calculation with the use of two optional optical blocking filters $(\mathrm{OBF})$ in the filter wheel (FW): a thin OBF ( $200 \mathrm{~nm}$ polyimide $+40 \mathrm{~nm} \mathrm{Al}$ ) to observe hot stars fainter than $\mathrm{m}_{\mathrm{V}} \sim 7.5$ with no significant degradation of energy resolution, and a thick OBF $(200 \mathrm{~nm}$ polyimide $+80 \mathrm{~nm} \mathrm{Al}$ ) to observe hot stars as bright as $\mathrm{m}_{\mathrm{v}}=2$.

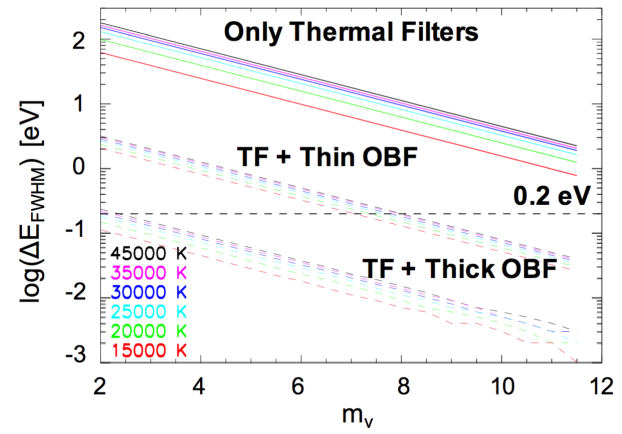

\section{Summary and Conclusions}

Fig. 4 X-IFU energy resolution degradation due to optical load from hot stars. The solid lines include UV/VIS stellar spectra attenuation by the Thermal Filters (TF) only, while the dashed lines include the attenuation with additional Optical Blocking Filters (OBF). Color figure online.

Thermal filters (TF) mounted on the cryostat aperture cylinder and FPA are needed to reduce the IR radiation onto the X-IFU detector array, to maintain the photon shot noise well below the nominal energy resolution, and to attenuate RF EMI onto the sensitive TES and SQUID read-out electronics.

Based on the heritage from previous missions we have chosen polyimide films coated with aluminum as the baseline materials for the TF. The currently investigated design consists of 5 separate filters, operating at different temperatures, for a total of $225 \mathrm{~nm}$ polyimide and $150 \mathrm{~nm}$ aluminum. Such configuration provides adequate attenuation of IR radiation. 
We plan to use metal meshes (open area $>95 \%$ ) for the two larger diameter filters to provide mechanical reinforcement, EMI RF attenuation, and, if needed, capability to warm up the filters for de-contamination. Currently investigated mesh consists of two layers of Ti alloy Ti6Al4V: 1) a structural layer with $5 \mathrm{~mm}$ pitch, $50 \mu \mathrm{m}$ bar width, $250 \mu \mathrm{m}$ thickness, and 2) an RF attenuator with $1 \mathrm{~mm}$ pitch, $10 \mu \mathrm{m}$ bar width, $30 \mu \mathrm{m}$ thickness. Preliminary structural analysis under a 10 mbar static load show a maximum tensile stress both on the mesh and on the attached polyimide film well within the ultimate tensile strengths of the adopted materials.

The optical load from the bright UV/VIS counterparts of X-ray sources (e.g. massive stars, AGN's, Mars) can degrade the X-IFU energy resolution by photon shot noise. We propose to use two optional optical blocking filters (OBF) on the filter wheel, namely: a thin OBF (200 nm polyimide $+40 \mathrm{~nm}$ $\mathrm{Al})$ to observe hot stars fainter than $\mathrm{m}_{\mathrm{V}} \sim 7.5$, and a thick OBF $(200 \mathrm{~nm}$ polyimide $+80 \mathrm{~nm} \mathrm{Al}$ ) to observe hot stars as bright as $\mathrm{m}_{\mathrm{V}}=2$.

A first set of filters will be procured in Q4 2015 to improve the TRL in the pre-assessment study phase. UV/VIS/IR transmission will be measured at different temperatures in the range $10-300 \mathrm{~K}$ to check the model. High spectral resolution $\mathrm{X}$-ray transmission measurements and X-ray photoelectron spectroscopy will be performed to model transmission near the absorption edges in the temperature range $80-300 \mathrm{~K}$ and to measure the surface aluminum oxide. Environmental tests will start in Q1-Q2, 2016.

Acknowledgements We acknowledge support by the Italian Space Agency (Contract number 2014-045-R.O.) and fruitful discussions and suggestions by LUXEL corp.

\section{References}

1. X. Barcons et al., J. Phys. Conf. Series, 610(1), 012008 (2015).

2. K. Nandra et al., eprint arXiv:1306.2307N (2013).

3. R. Willingale et al., eprint arXiv:1307.1709W (2013).

4. L. Ravera et al., Proc. SPIE, 9144, 9144-2L (2014).

5. M. Barbera et al., Proc. SPIE, 9144, 9144-5U (2014).

6. D. McCammon et al., JLTP, 151, Issue 3-4, 715-720 (2008).

7. M.D. Audley et al., Proc. SPIE, 3765, 751-761 (1999).

8. R.L. Kelley et al., PASJ, 59, 77-112 (2007).

9. C.P. de Vries et al., Proc. SPIE, 7732, 7732-13 (2010).

10. T. Takahashi et al., Proc. SPIE, 7732, 7732-0Z (2010).

11. M. Barbera et al., Proc. SPIE, 4851, 264-269 (2003).

12. M. Barbera et al., Proc. SPIE, 8859, 8859-14 (2013).

13. S. Sciortino et al., eprint arXiv:1306.2333 (2013). 